\title{
Prognostic value of procalcitonin in community-acquired pneumonia
}

\author{
P. Schuetz*, ${ }^{*}$ I. Suter-Widmer ${ }^{\#, f}$, A. Chaudri"\#,f, M. Christ-Crain\#, W. Zimmerlii and \\ B. Mueller* ${ }^{*}$ for the Procalcitonin-Guided Antibiotic Therapy and Hospitalisation in \\ Patients with Lower Respiratory Tract Infections (ProHOSP) Study Group ${ }^{\S}$
}

ABSTRACT: The prognostic value of procalcitonin (PCT) levels to predict mortality and other adverse events in community-acquired pneumonia (CAP) remains undefined.

We assessed the performance of PCT overall, stratified into four predefined procalcitonin tiers $\left(<0.1,0.1-0.25,>0.25-0.5,>0.5 \mu \mathrm{g} \cdot \mathrm{L}^{-1}\right)$ and stratified by Pneumonia Severity Index (PSI) and CURB-65 (confusion, urea $>7 \mathrm{mmol} \cdot \mathrm{L}^{-1}$, respiratory frequency $\geqslant 30$ breaths $\cdot \mathrm{min}^{-1}$, systolic blood pressure $<90 \mathrm{mmHg}$ or diastolic blood pressure $\leqslant 60 \mathrm{mmHg}$, and age $\geqslant 65$ yrs) risk classes to predict all-cause mortality and adverse events within 30 days follow-up in 925 CAP patients.

In receiver operating characteristic curves, initial PCT levels performed only moderately for mortality prediction (area under the curve (AUC) 0.60 ) and did not improve clinical risk scores. Follow-up measurements on days 3, 5 and 7 showed better prognostic performance (AUCs 0.61, 0.68 and 0.73 ). For prediction of adverse events, the AUC was 0.66 and PCT significantly improved the PSI (from 0.67 to 0.71) and the CURB-65 (from 0.64 to 0.70). In Kaplan-Meier curves, PCT tiers significantly separated patients within PSI and CURB-65 risk classes for adverse events prediction, but not for mortality. Reclassification analysis confirmed the added value of PCT for adverse event prediction, but not mortality.

Initial PCT levels provide only moderate prognostic information concerning mortality risk and did not improve clinical risk scores. However, PCT was helpful during follow-up and for prediction of adverse events and, thereby, improved the PSI and CURB65 scores.

KEYWORDS: CURB65, mortality, pneumonia, Pneumonia Severity Index, procalcitonin

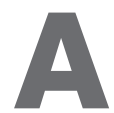
ssessment of disease severity and prediction of outcome are prerequisites for adequate allocation of healthcare resources and therapeutic options in the management of community-acquired pneumonia (CAP). This includes decisions regarding hospital or intensive care admission, diagnostic work-up, route of antimicrobial therapy and evaluation for early discharge. To optimise and to reduce unnecessary hospital admission rates, professional organisations have developed prediction rules and propagated guidelines to stratify patients with CAP based on predicted risk for mortality $[1,2]$. The Pneumonia Severity Index (PSI) is a well-validated scoring system that assesses the risk of death in a two-step algorithm [3]. The tool was developed to identify patient at low risk for mortality [3]. However, it is complex and strongly dependent on age, limiting its general implementation in routine care. The less complex CURB65 (confusion, urea $>7 \mathrm{mmol} \cdot \mathrm{L}^{-1}$ respiratory frequency $\geqslant 30$ breaths $\cdot \mathrm{min}^{-1}$, low blood pressure (systolic value $<90 \mathrm{mmHg}$ or diastolic value $\leqslant 60 \mathrm{mmHg}$ ) and age $\geqslant 65 \mathrm{yrs}$ ) score, focuses on five predictors [4]. This score is easier to calculate, but has a slightly inferior prognostic accuracy. Both risk scores were validated for the prediction of mortality only, and their ability to predict other CAP-associated adverse outcomes is not validated. Both scores have limitations for clinical use, including practicability, risk of miscalibration, and only moderate sensitivity and specificity, which leads to hospitalisation of patients where outpatient treatment would have been preferable [5]. Thus, additional risk factors and prognostic biomarkers potentially enhance the prognostic performance of these established risk scores in CAP patients.

Previous studies investigated the prognostic potential of procalcitonin (PCT) for mortality prediction [6]. While studies in the intensive care unit (ICU) setting $[7,8]$ and in high-risk patients
AFFILIATIONS

*Harvard School of Public Health, Boston, MA, USA.

\#Division of Endocrinology, Diabetes and Clinical Nutrition, Dept of Internal Medicine, University Hospital Basel, Basel, and

'Medical University Clinic, Medical Faculty, University of Basel, ${ }^{+}$Dept of Internal Medicine, Kantonsspital Aarau, Aarau, Switzerland.

${ }^{\S} \mathrm{A}$ full list of the members of the ProHOSP Study Group and their affiliations can be found in the Acknowledgements section. ${ }^{f}$ These authors contributed equally to the study.

CORRESPONDENCE

P. Schuetz

Harvard School of Public Health

677 Huntington Avenue

Boston

MA 02115

USA

E-mail: Schuetzph@gmail.com

Received:

March 052010

Accepted after revision:

June 142010

First published online:

July 012010 
[9] have demonstrated a high prognostic accuracy of PCT, results from studies in the emergency department (ED) with lower severity patients did not show consistent results [9-11]. A study from the German Community-Acquired pneumonia Competence Network (CAPNETZ) recently found that PCT levels on admission improve the prognostic performance of the CRB-65 (confusion, respiratory rate $\geqslant 30$ breaths $\cdot \mathrm{min}^{-1}$, low blood pressure (systolic value $<90 \mathrm{mmHg}$ or diastolic value $\leqslant 60 \mathrm{mmHg}$ ) and age $\geqslant 65 \mathrm{yrs}$ ) score [11]. However, a large USA-based CAP study found only a moderate additive value of PCT when compared to the PSI and CURB-65 scores [12].

The aim of the present study was to validate these previous findings in a large, well-defined cohort of CAP patients enrolled in the multicentre Procalcitonin-Guided Antibiotic Therapy and Hospitalisation in Patients with Lower Respiratory Tract Infections (ProHOSP) study [13], and to evaluate the prognostic potential of initial and serial PCT levels for prediction of mortality and other adverse events.

\section{METHODS}

\section{Study design and setting}

As a predefined ancillary project, we used clinical data and PCT levels from all patients with CAP enrolled in the multicenter ProHOSP study [13]. The design of the ProHOSP study has been reported in detail elsewhere [13]. Briefly, from October 2006 to March 2008, a total of 1,359 consecutive patients with presumed lower respiratory tract infection (LRTI) from six different hospitals located in the northern part of Switzerland were included; 925 patients had a definite diagnosis of CAP, and the remaining patients suffered from chronic obstructive pulmonary disease exacerbation and acute bronchitis. Patients were randomly assigned to an intervention group, where guidance of antibiotic therapy was based on PCT cut-off ranges, or a standard group, where guidance of antibiotic therapy was based on enforced guideline recommendations without knowledge of PCT. The primary endpoint of the study was to compare the risk for adverse medical outcomes within 30 days following the ED admission by PCT guidance when compared with standard recommended guidelines. A predefined secondary end-point was to investigate the prognostic potential of PCT in CAP patients.

\section{Selection of participants}

Patients $>18$ yrs of age with a suspected LRTI as their principal diagnosis on admission were eligible for the ProHOSP study. In accordance with guidelines, LRTI was defined by the presence of at least one respiratory symptom (cough, sputum production, dyspnoea, tachypnoea or pleuritic pain) plus at least one finding during auscultation (rales or crepitation), or one sign of infection (core body temperature $>38.0^{\circ} \mathrm{C}$, shivering, leukocyte count $>1 \times 10^{10}$ cells $\cdot \mathrm{L}^{-1}$ or $<4 \times 10^{9}$ cells $\cdot \mathrm{L}^{-1}$ ) independent of antibiotic pre-treatment. CAP was defined as a new infiltrate on chest radiograph [1, 14]. Patients were examined on admission to the ED by a resident physician supervised by a board-certified specialist in internal medicine. The standardised baseline assessment included medical history, clinical examination, lab tests and chest radiography. For all patients with CAP, the PSI and the CURB-65 score were calculated on admission as described elsewhere $[3,4]$. The study protocol was approved by all local ethical committees, and written informed consent was obtained from all participants.

\section{PCT measurement}

PCT was measured with a highly sensitive, time-resolved amplified cryptate emission technology assay (PCT Kryptor ${ }^{\circledR}$; BRAHMS AG, Hennigsdorf, Germany). The assay has a detection limit of $0.02 \mu \mathrm{g} \cdot \mathrm{L}^{-1}$ and functional assay sensitivity of $0.06 \mu \mathrm{g} \cdot \mathrm{L}^{-1}$, i.e. $3-10$-fold over normal mean values.

\section{Study end-points}

The primary end-point for this analysis was all-cause mortality within 30 days following randomisation; the secondary endpoints were adverse events, defined as death, ICU admission or any disease-specific complications (including empyema, abscess and acute respiratory distress syndrome) within

\begin{tabular}{|c|c|}
\hline Characteristic & Baseline value \\
\hline \multicolumn{2}{|l|}{ Demographic characteristics } \\
\hline Age yrs & $73(58-82)$ \\
\hline Males & $544(59)$ \\
\hline \multicolumn{2}{|l|}{ Coexisting illnesses } \\
\hline Chronic heart failure & $159(17)$ \\
\hline Renal failure & $206(22)$ \\
\hline COPD & $282(30)$ \\
\hline \multicolumn{2}{|l|}{ Clinical findings } \\
\hline Confusion & $74(9)$ \\
\hline Respiratory rate breaths $\cdot \mathrm{min}^{-1}$ & $20(16-25)$ \\
\hline Systolic blood pressure $\mathrm{mmHg}$ & $132(119-148)$ \\
\hline Heart rate beats $\cdot \mathrm{min}^{-1}$ & $95(82-108)$ \\
\hline Body temperature ${ }^{\circ} \mathrm{C}$ & $38.1(37.2-38.9)$ \\
\hline PSI points & $92(68-116)$ \\
\hline \multicolumn{2}{|l|}{ PSI class } \\
\hline I-III & $452(49)$ \\
\hline IV & 349 (38) \\
\hline V & $124(13)$ \\
\hline CURB-65 points & $2(1-2)$ \\
\hline \multicolumn{2}{|l|}{ CURB class } \\
\hline $0-1$ & $459(50)$ \\
\hline 2 & $306(33)$ \\
\hline $3-5$ & $160(17)$ \\
\hline \multicolumn{2}{|l|}{ Follow-up } \\
\hline Outpatient treatment & $52(6)$ \\
\hline Length of stay in hospitalised patients days & $8(5-13)$ \\
\hline \multicolumn{2}{|l|}{ 30-day outcomes } \\
\hline Any adverse events & $134(14.5)$ \\
\hline All-cause mortality & $50(5.4)$ \\
\hline ICU admission & $83(9.0)$ \\
\hline Disease-specific complications & $31(3.4)$ \\
\hline
\end{tabular}

Data are presented as median (interquartile range) or $\mathrm{n}(\%)$. COPD: chronic obstructive pulmonary disease; PSI: Pneumonia Severity Index; CURB-65 confusion, urea $>7 \mathrm{mmol} \cdot \mathrm{L}^{-1}$, respiratory frequency $\geqslant 30$ breaths $\cdot \mathrm{min}^{-1}$, low blood pressure (systolic value $<90 \mathrm{mmHg}$ or diastolic value $\leqslant 60 \mathrm{mmHg}$ ) and age $\geqslant 65$ yrs; ICU: intensive care unit. ${ }^{*}: \mathrm{n}=925$. 
30 days. Outcome assessment was standardised and monitored by an independent Safety and Monitoring Board, consisting of three specialists in pneumology, infectious diseases and intensive care medicine as part of the ProHOSP protocol [13]. Outcome was assessed during hospital stay at days 3, 5 and 7, at hospital discharge, and by structured telephone interviews at days 30 by blinded medical students. In the case that the patient indicated any complication during or following hospital discharge or was unable to give adequate information, the interviewer was obliged to contact the treating general practitioner or hospital to receive notification of the prescription, or a copy of the hospital transfer or discharge letter.

\section{Statistical analysis}

First, we assessed the prognostic accuracy of initial PCT levels to predict primary and secondary end-points by plotting receiver operating characterstic (ROC) curves. The area under the ROC curve (AUC) is a summary measure of criteria and cut-off point choices. We report sensitivity, specificity, and positive and negative likelihood ratios at different cut-off points. We then assessed whether PCT significantly increased within four PCT cut-off ranges $\left(<0.1,0.1-0.25,>0.25-0.50\right.$ and $\left.>0.50 \mu \mathrm{g} \cdot \mathrm{L}^{-1}\right)$ with Kruskal-Wallis one-way ANOVA for multigroup comparisons. These cut-offs were chosen because they are the basis of the clinical algorithm for antibiotic stewardship [15].
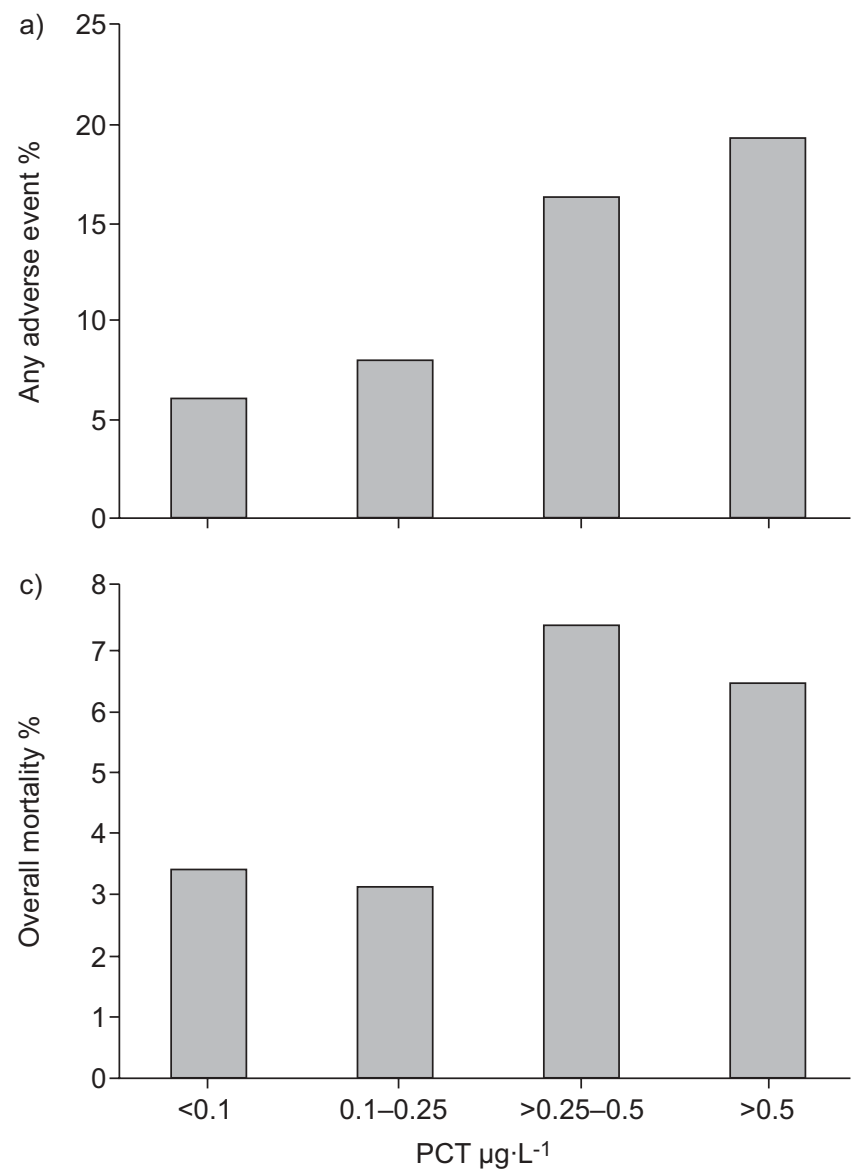

Secondly, we tested whether PCT improves the performance of the PSI and CURB-65 scores by comparing ROC curves of the joint logistic regression of PCT and each of the risk scores to ROC curves limited to the scores only. Thereby, the scores were included as PSI classes (1-5) and CURB-65 classes (1-5) into the model. We also plotted Kaplan-Meier survival curves, and stratified patients by PCT cut-off points overall and in $a$ priori PSI and CURB-65 risk categories; thereby, low-risk patients were defined as PSI classes I-III and CURB65 classes $0-1$, and high-risk patients were defined as PSI classes IV-V and CURB-65 classes 2-5. We tested for differences between PCT tiers with log rank trend tests.

To investigate the potential clinical usefulness of adding PCT to clinical risk scores, we further calculated reclassification tables as proposed by PENCINA et al. [16]. For estimating meaningful a priori risk categories, we used predicted probabilities for 30-day mortality from the original PSI and CURB65 cohorts $[3,4,17]$. For prediction of adverse events, we used the predicted risks, as found in the present study, for the PSI and CURB-65 classes.

Finally, we analysed follow-up PCT measurement by plotting crude values, and the difference between initial levels on admission, and day-3, -5 and -7 values in survivors and nonsurvivors.
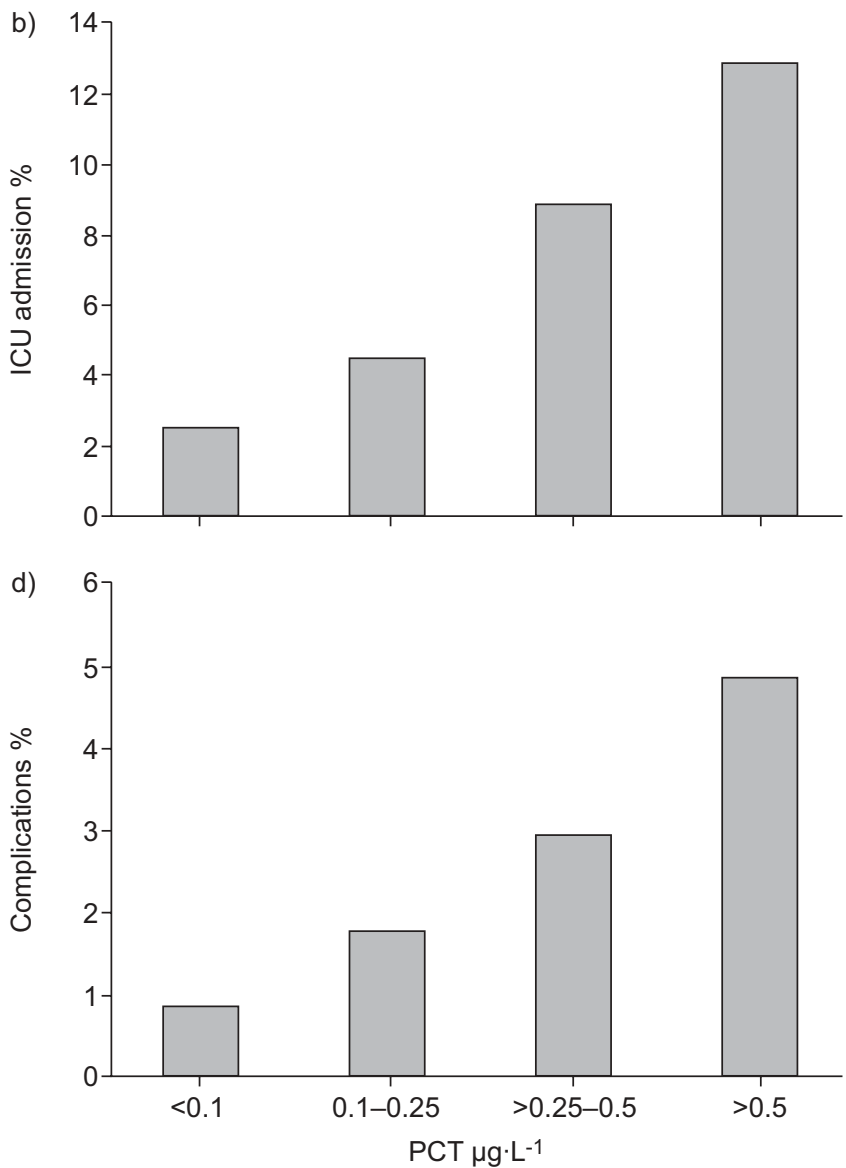

FIGURE 1. Risk of a) any adverse event $(p<0.001)$, b) intensive care unit $(I C U)$ admission $(p<0.001)$, c) overall mortality $(p=0.15)$ and $d)$ complications $(p=0.06)$ in different procalcitonin (PCT) cut-offs. 
All testing was two-tailed and p-values $<0.05$ were considered to indicate statistical significance. All calculations were performed using STATA 9.2 (StataCorp, College Station, TX, USA).

\section{RESULTS}

\section{Patient population}

A total of 925 persons with a definite diagnosis of CAP were included in the analysis. In one patient, initial PCT was not available. The median age of patients at the time of study enrolment was 73 yrs and $41 \%$ were females. The number of patients with CAP in the six participating centres ranged 122 210 . Around $50 \%$ of patients were classified as high-risk by PSI score (classes IV-V) and CURB-65 score (classes 2-5).

Overall, $14.5 \%$ of patients experienced an adverse event within 30 days following inclusion, including death (5.4\%) and ICU admission (9.0\%). Disease-specific complications occurred in $3.4 \%$ and all were empyema. Notably, 30 patients had more than one adverse event, particularly ICU admission and empyema $(n=12)$, and ICU admission and subsequent death $(n=15)$. Baseline characteristics in all CAP patients are presented in table 1.

\section{Prognostic accuracy of PCT to predict outcome}

Nonsurvivors had significantly increased median PCT levels on admission compared to survivors $\left(0.83 \mu \mathrm{g} \cdot \mathrm{L}^{-1}\right.$ (interquartile range (IQR) $0.30-5.67 \mu \mathrm{g} \cdot \mathrm{L}^{-1}$ ) versus $0.44 \mu \mathrm{g} \cdot \mathrm{L}^{-1}$ (IQR $0.15-$ $\left.\left.2.63 \mu \mathrm{g} \cdot \mathrm{L}^{-1}\right) ; \mathrm{p}=0.02\right)$. This was also true for patients with adverse events compared to patients without adverse events $\left(1.30 \mu \mathrm{g} \cdot \mathrm{L}^{-1} \quad\right.$ (IQR $\left.\quad 0.38-7.47 \mu \mathrm{g} \cdot \mathrm{L}^{-1}\right)$ versus $0.39 \mu \mathrm{g} \cdot \mathrm{L}^{-1} \quad(0.14-$ $\left.\left.2.2 \mu \mathrm{g} \cdot \mathrm{L}^{-1}\right) ; \mathrm{p}<0.001\right)$. When stratifying patients according to initial PCT levels, 12.7, 24.2, 14.6 and $48.5 \%$ were in PCT tiers $<0.1,0.1-0.25,>0.25-0.5$ and $>0.5 \mu \mathrm{g} \cdot \mathrm{L}^{-1}$. The risk of any adverse event and ICU admission strongly increased with higher PCT tiers (fig. 1); the increase was not significant for death and disease-related complications.

In ROC curve analysis for prediction of 30-day mortality, initial PCT had an AUC of 0.60, which was significantly lower compared to the PSI and CURB-65 scores (table 2). When combining PCT and each one of the two risk scores in a logistic regression model, PCT did not significantly improve either of the two scores for mortality prediction. For prediction of adverse events, initial PCT levels had an AUC of 0.66, which was in the range of the PSI (AUC 0.67) and the CURB-65 (AUC 0.64) score. Combination of PCT to each of the scores significantly improved their prognostic ability to predict adverse events. For prediction of ICU admission, PCT was in the range of both scores and improved their prognostic ability significantly. The peak PCT level had significantly higher AUCs for ICU prediction compared to both scores. For prediction of disease-specific complications, initial and peak PCT had a significantly higher accuracy as compared to the PSI and CURB-65 score, and initial PCT levels significantly improved both scores.

Table 3 shows sensitivity, specificity, and positive and negative likelihood ratios for different PCT cut-off values overall, and in low- and high-risk PSI patients. Notably, in high-risk patients, PCT had lower sensitivity and specificity at all different cut-offs for mortality prediction compared with prediction of adverse outcomes. 


\begin{tabular}{|c|c|c|c|c|c|c|c|c|}
\hline \multirow{2}{*}{ TABLE 3} & \multicolumn{4}{|c|}{ Mortality prediction } & \multicolumn{4}{|c|}{ Adverse event prediction } \\
\hline & Sensitivity \% & Specificity \% & Positive LR & Negative LR & Sensitivity \% & Specificity \% & Positive LR & Negative LR \\
\hline \multicolumn{9}{|l|}{ All CAP patients $\#$} \\
\hline PCT $>0.5 \mu \mathrm{g} \cdot \mathrm{L}^{-1}$ & 58.00 & 51.95 & 1.21 & 0.81 & 64.93 & 54.18 & 1.42 & 0.65 \\
\hline PCT $>1.0 \mu \mathrm{g} \cdot \mathrm{L}^{-1}$ & 48.00 & 61.10 & 1.23 & 0.85 & 55.22 & 63.29 & 1.50 & 0.71 \\
\hline PCT $>5.0 \mu \mathrm{g} \cdot \mathrm{L}^{-1}$ & 26.00 & 83.75 & 1.60 & 0.88 & 31.34 & 85.70 & 2.19 & 0.80 \\
\hline \multicolumn{9}{|l|}{ PSI low risk } \\
\hline РCT $>0.1 \mu \mathrm{g} \cdot \mathrm{L}^{-1}$ & 100.00 & 19.11 & 1.24 & 0.00 & 94.12 & 20.10 & 1.18 & 0.29 \\
\hline \multicolumn{9}{|l|}{ PSI high risk ${ }^{+}$} \\
\hline PCT $>0.1 \mu \mathrm{g} \cdot \mathrm{L}^{-1}$ & 93.75 & 5.90 & 1.00 & 1.06 & 96.00 & 6.45 & 1.03 & 0.62 \\
\hline PCT $>0.25 \mu \mathrm{g} \cdot \mathrm{L}^{-1}$ & 79.17 & 28.30 & 1.10 & 0.74 & 82.00 & 30.11 & 1.17 & 0.60 \\
\hline $\mathrm{PCT}>0.5 \mu \mathrm{g} \cdot \mathrm{L}^{-1}$ & 60.42 & 45.05 & 1.10 & 0.88 & 65.00 & 47.04 & 1.23 & 0.74 \\
\hline PCT $>1.0 \mu \mathrm{g} \cdot \mathrm{L}^{-1}$ & 50.00 & 55.66 & 1.13 & 0.90 & 56.00 & 58.06 & 1.34 & 0.76 \\
\hline PCT $>5.0 \mu \mathrm{g} \cdot \mathrm{L}^{-1}$ & 27.08 & 82.55 & 1.55 & 0.88 & 32.00 & 85.22 & 2.16 & 0.80 \\
\hline
\end{tabular}

CAP: community-acquired pneumonia; PSI: Pneumonia Severity Index; PCT: procalcitonin. ${ }^{*}: \mathrm{n}=924 ;{ }^{\bullet}:$ PSI I-III, $\mathrm{n}=452 ;{ }^{+}:$PSI IV-V, $\mathrm{n}=472$

\section{Death and adverse events in different PCT cut-off ranges}

Figure 2 shows the Kaplan-Meier curves according to the different PCT tiers overall (upper panel), and in PSI low(middle panel) and high-risk patients (lower panel). Overall, patients in the lowest two PCT tiers had lower mortality rates as compared to the higher tiers $(p=0.05)$. However, in a subgroup of PSI low- and high-risk patients, PCT tiers did not significantly separate patients. Concerning adverse events overall, the risk significantly increased about three-fold, from $6 \%$ in the lowest PCT tier to $19 \%$ in the highest tier $(\mathrm{p}<0.001)$. PCT tiers adequately separated patients with and without adverse events in low risk patients $(p=0.01)$, but not in high risk patients $(p=0.07)$.

The same analysis for low- and high-risk CURB-65 categories showed that PCT significantly separated patients with and without adverse events in low- $(p<0.001)$ and high-risk $(\mathrm{p}=0.006)$ CURB-65 classes; however, there was no significant separation for mortality $(p=0.27$ and 0.79$)$ (see online supplementary Figure 1).

\section{Net reclassification improvement}

We calculated in-sample reclassification behaviour for survivors and nonsurvivors, and for patients who did or did not experience an adverse event. For mortality prediction, reclassification tables showed that addition of PCT improved risk prediction of the PSI for only one nonsurvivor. For adverse events prediction, 12 patients with adverse events were classified more accurately with the combined model of PCT and the PSI compared with the PSI alone. Table 4 shows the reclassification table for adverse events of patients classified with the PSI score (vertical) compared with the combined model of PSI score and PCT level (horizontal).
Repeating the analysis with the CURB-65 score instead of PSI showed similar results. For only one nonsurvivor and 21 patients with an adverse event, reclassification was more accurate when the model with PCT was used.

\section{Follow-up PCT measurements}

Figure 3 shows absolute PCT levels and relative changes on admission and at days 3,5 and 7, comparing survivors and nonsurvivors. Nonsurvivors had significantly increased PCT concentrations on each of the follow-up days compared with survivors. When analysing relative changes in PCT concentrations between admission and days 3, 5 and 7, nonsurvivors had a small relative increase on day 3 , while survivors had a decrease from admission to day 3. Similarly, nonsurvivors had a significantly less pronounced decrease on days 5 and 7 compared to nonsurvivors. ROC analysis revealed AUCs of PCT for discrimination of survivors from nonsurvivors on days 3, 5 and 7 of 0.61 (95\% CI 0.51-0.70), 0.68 (95\% CI 0.59 $0.77)$ and 0.73 (95\% CI 0.64-0.84), respectively.

In patients with adverse events, PCT was significantly increased on all follow-up days ( $\mathrm{p}<0.01$ for each comparison) compared to patients without adverse events. AUCs for discrimination of patients with and without adverse events on days 3, 5 and 7 were 0.67 (95\% CI 0.61-0.72), 0.71 (95\% CI $0.65-0.76)$ and 0.68 (95\% CI $0.62-0.74)$, respectively.

\section{DISCUSSION}

Within this large-scale, multicentre study of well-defined CAP patients, PCT levels on admission had an only moderate prognostic ability to predict 30-day mortality, and did not significantly improve the PSI and CURB-65 scores in combined 
a)

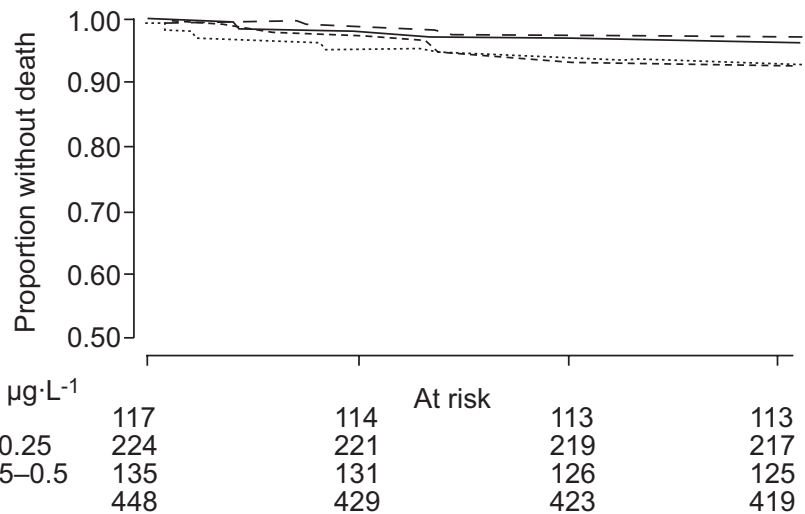

c)

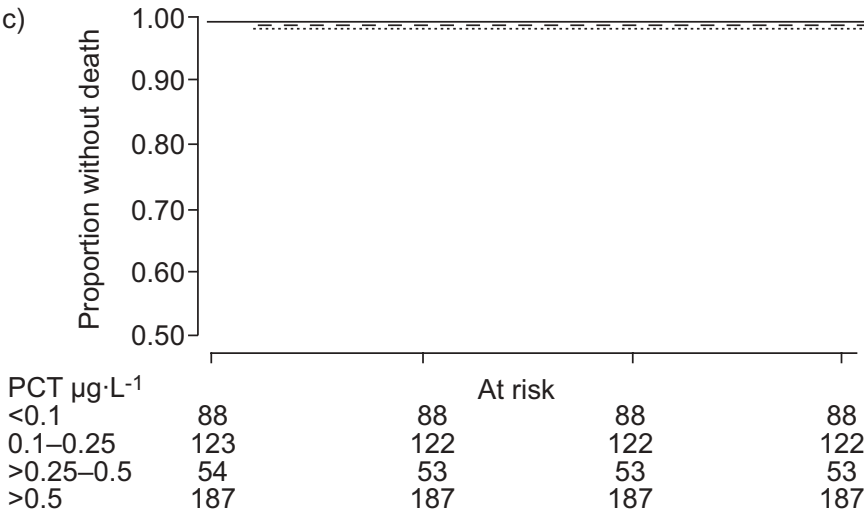

e)

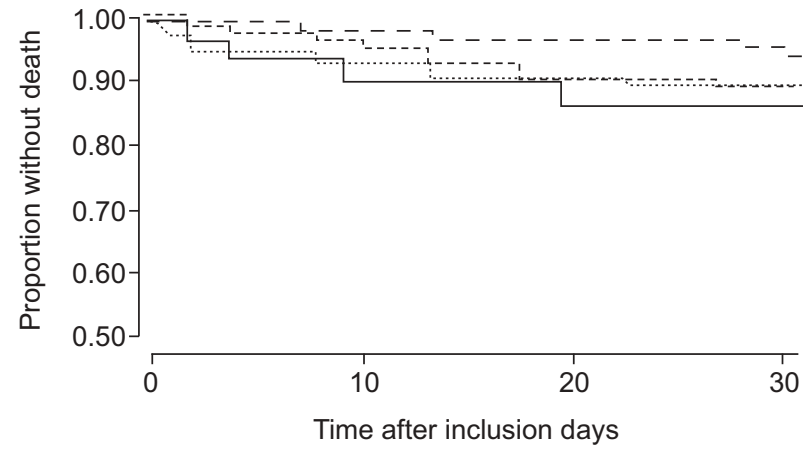

PCT $\mu g \cdot L^{-1}$

$<0.1 \quad 29$

$\begin{array}{ll}0.1-0.25 & 101\end{array}$

$>0.25-0.5 \quad 81$

$>0.5-261$
At risk

$\begin{array}{ccc}26 & & \\ 99 & 25 & 25 \\ 78 & 97 & 95 \\ 242 & 73 & 72 \\ & 236 & 232\end{array}$

b)

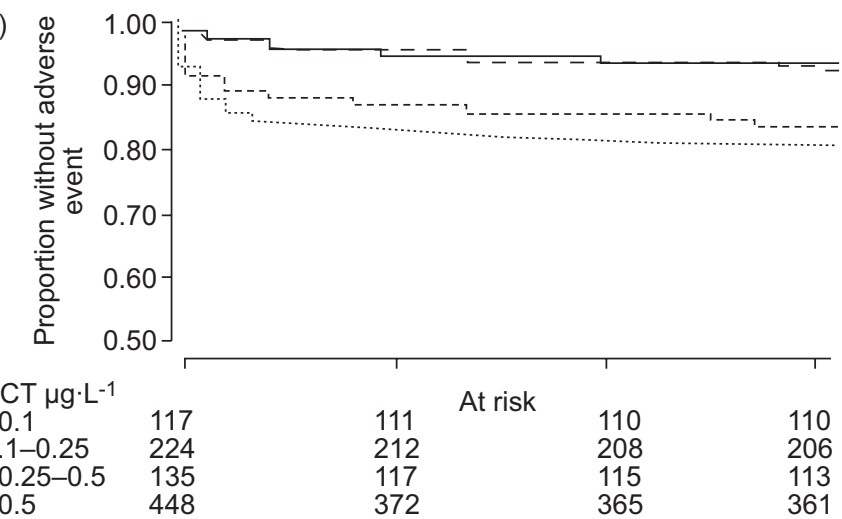

d)

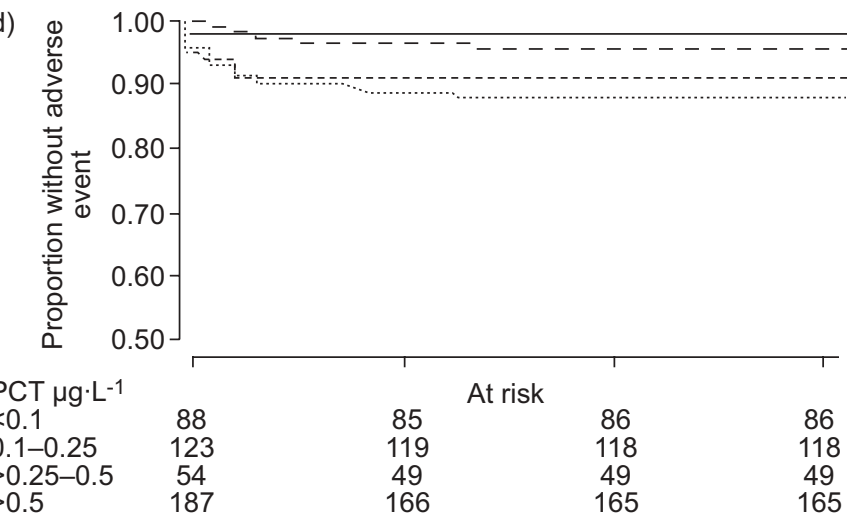

f)

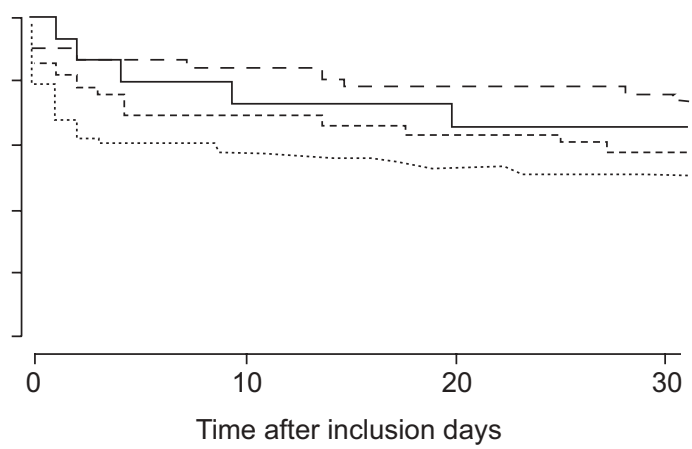

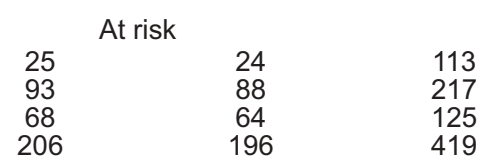

FIGURE 2. Kaplan-Meier plots of $a, b)$ overall, and c, d) low- (Pneumonia Severity Index (PSI) class I-III) and e, f) high-risk (PSI class IV-V) patients stratified by

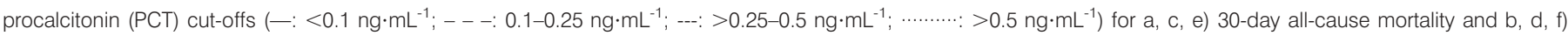
adverse events. Log rank p-values were a) 0.05 , b) $<0.0001$, c) 0.25 , d) 0.01 , e) 0.42 and f) 0.07 .

logistic regression analysis and reclassification tables. However, follow up PCT measurements showed a better prognostic performance. In addition, we found that PCT was a significant predictor for serious CAP-associated adverse events and, thereby, improved the accuracy of the PSI as well as the CURB-65 scores.

PCT has emerged as a diagnostic biomarker for estimating the likelihood of a bacterial infection requiring immediate antibiotic therapy in CAP and suspected sepsis. Clinical trials from different settings and different countries have established that PCT can be safely used to decide upon initiation and duration of antibiotic therapy and, thereby, counteract antibiotic overuse and the resulting risks of side effects and emerging bacterial multi-resistance [18-22]. The prognostic value of PCT, however, is less clear. JENSEN et al. [7] found that, in the ICU setting, a high maximum PCT level and a PCT increase for 1 day were independent predictors of 90-day all-cause mortality. We recently found a high prognostic accuracy of initial and serial PCT levels in patients with Legionella CAP for 
TABLE 4 Reclassification of Pneumonia Severity Index (PSI) for prediction of adverse events

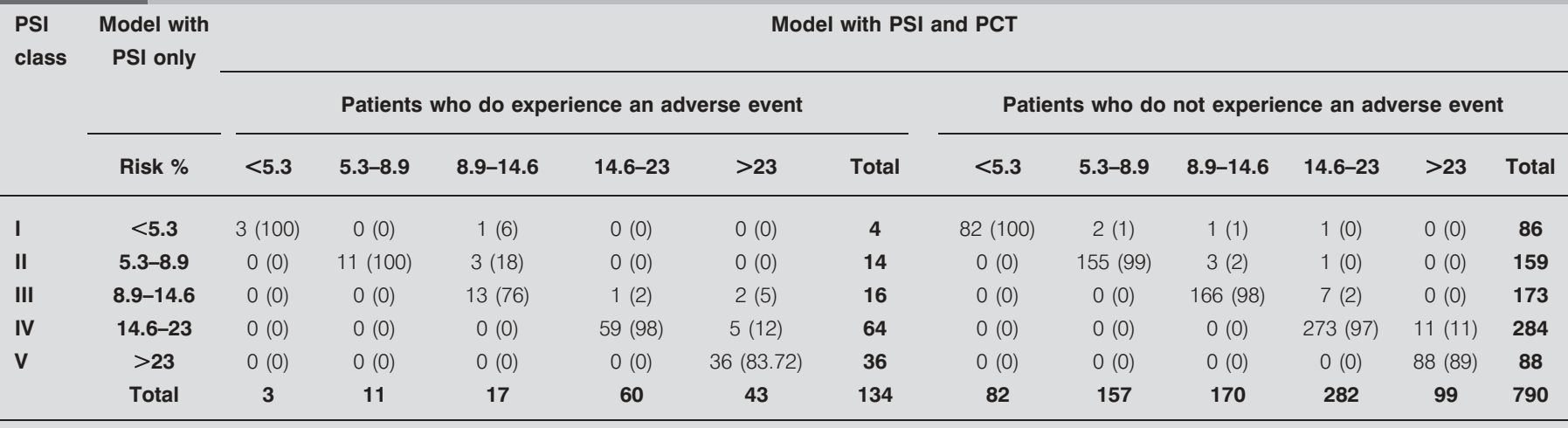

Data are presented as $n(\%)$.

prediction of mortality and need for ICU admission [9]. A study from the German CAPNETZ reported a high prognostic accuracy of PCT to predict mortality with a AUC of 0.80 in a mixed in- and outpatient setting [11]. In contrast to these findings, a secondary analysis of the Genetic and Inflammatory Markers of Sepsis (GenIMS) cohort reported an only moderate additional value of PCT when compared to the PSI and CURB65 scores [12]. Within this study, low PCT levels of $<0.1 \mu \mathrm{g} \cdot \mathrm{L}^{-1}$ ruled out mortality even in high-risk CAP patients, as assessed by clinical scores. Finally, a Spanish study found that Creactive protein, but not PCT or interleukins, improved clinical scores for mortality prediction [23]. Our data do not support the use of admission PCT for mortality prediction in CAP, because initial PCT per se had an only moderate prognostic value and did not improve the PSI and CURB-65 scores. The fact that patients with low PCT levels still had fatal outcomes within our study may have many different reasons. First, we assessed all-cause mortality, and some patients may have died in the absence of severe infection because of advanced age and severe comorbidities. The PSI score is largely age-driven and incorporates comorbidities, which increases its performance to predict all cause mortality within a 30 days follow-up. Secondly, death occurred equally within the 30 days followup. Thus it is possible that secondary complications or aggravation of initially uncomplicated CAP may be missed by initial PCT measurement. It has previously been suggested that serial PCT measurement may help to assess resolution of CAP. MENENDEZ et al. [24] reported that decreasing PCT levels at $72 \mathrm{~h}$ in addition to clinical criteria improved the prediction of absence of severe complications. Similarly, another Spanish study found that higher follow-up PCT concentrations were associated with development of complications and death [25]. Our trial confirms these findings and showed that follow-up measurements of PCT improved its prognostic accuracy, particularly for prediction of mortality: nonsurvivors showed a different kinetic compared with survivors, with a relative increase of PCT levels from day 0 to day 3, and a less pronounced PCT decrease thereafter. Thus, based on our findings and these previous studies, it is more helpful to consider the dynamics of PCT levels in CAP and not rely on initial levels.

We found a high prognostic accuracy of PCT for prediction of CAP-associated serious adverse events, particularly the need for ICU admission and disease-related complications. Thereby, PCT had a similar prognostic accuracy compared to the PSI and CURB-65 scores, and improved their discriminatory ability in logistic regression models and in clinical reclassification
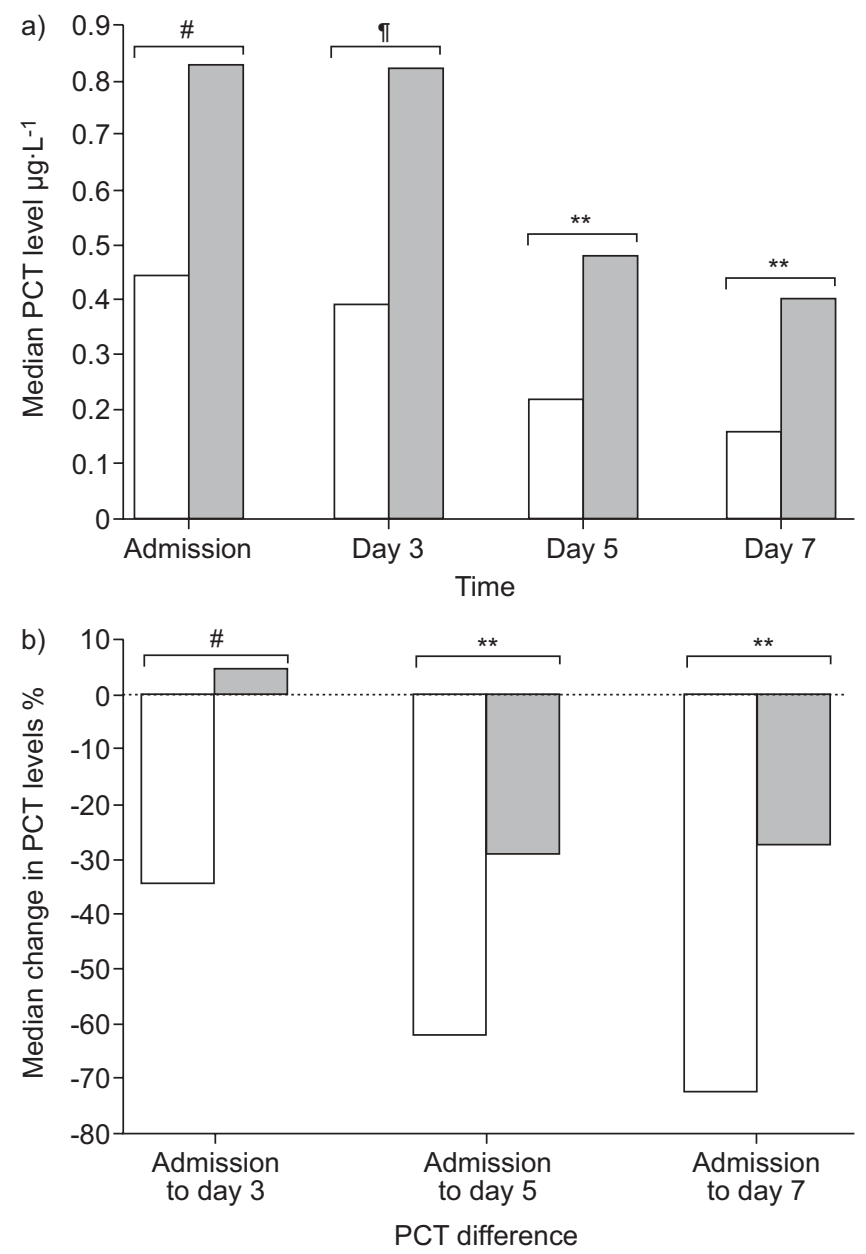

FIGURE 3. Serial procalcitonin (PCT) measurements in survivors and nonsurvivors. a) absolute median PCT values on admission and days 3, 5 and 7 . b) relative changes in PCT concentration compared with baseline values on admission. $\square$ : survivors; $\square$ : nonsurvivors. ${ }^{\#}: p=0.02 ; "$ : $p=0.03$; ${ }^{* *}: p<0.01$. 
analysis. ICU admission in CAP patients is mostly related to either severe sepsis and need for early resuscitation or respiratory failure due to severe CAP. One may argue that mortality is a more objective outcome and outcomes like ICU admission or disease-specific complications are prone to interobserver variability. However, within the ProHOSP study, we provided guidelines [13] for ICU admission and for diagnosis of empyema, and all outcomes were assessed by an independent monitoring board. Nonmortality endpoints may be of particular importance in CAP when evaluating tools for guiding site-of-care decisions. PSI and CURB-65 scores were developed to predict short-term mortality, and their lower prognostic accuracy for nonmortality end-points is, thus, not surprising. The scores may underestimate the risk for complications, especially in younger patients with severe illness. HuANG et al. [12] reported that adding PCT to the assessment of high-risk patients significantly improved the ability to rule out the likelihood of death. We found this to be true for other CAP-associated complications. Conversely, we found that high PCT levels point to higher risk for adverse events within low-risk PSI or CURB-65 patients.

This study has limitations. Exclusion of patients with dementia, immunosuppression, concomitant infections and active intravenous drug abuse limits its generalisability. We used 30day all-cause mortality, in line with the original PSI and CURB65 reports [3, 4], and it is possible that PCT would perform better if only sepsis-related mortality was considered. We previously found a high positive correlation of PCT and positive blood culture results [26], but because of limited sample size, we did not assess how PCT performs in subgroups of microbiologically confirmed CAP patients. We used ICU admission as part of our combined end-point and indications for ICU admission may vary between physicians, hospitals and countries. Importantly, with an observational design, this study can not address whether PCT measurement will improve risk scores for adverse event prediction in clinical practice and also whether adding PCT to clinical scores would be cost-effective. Some patients with uncomplicated resolution of CAP in the hospital setting could develop complications if outpatient treatment would have been chosen. Therefore, prospective intervention studies need to be conducted to investigate whether PCT really adds useful prognostic information and, thereby, improves the daily clinical management of patients with CAP.

In conclusion, this study confirms the predictive value of PCT in combination with PSI or CURB-65 in regard to serious adverse events in adult CAP patients, and much less for mortality prediction. Future studies must address whether adding PCT to risk scores can increase their safe implementation in clinical practice.

\section{STATEMENT OF INTEREST}

Statements of interest for P. Schuetz, M. Christ-Crain and B. Mueller can be found at www.erj.ersjournals.com/site/misc/statements.xhtml

\section{ACKNOWLEDGEMENTS}

We are grateful to the Data Safety and Monitoring Board, namely A.P. Perruchoud, S.Harbarth and A.Azzola, for continuous supervision of this trial, and all local physicians, the nursing staff, the patients and their relatives who participated in this study. Especially, we thank the staff of the ED, medical clinics and central laboratories of the University Hospital Basel (Basel, Switzerland), the Cantonal Hospitals Liestal, Aarau, Luzern and Muensterlingen and the "Buergerspital" Solothurn (all Switzerland) for their very helpful assistance, patience and technical support. We thank other members of the Procalcitonin Guided Antibiotic Therapy and Hospitalisation in Patients with Lower Respiratory Tract Infections (ProHOSP) Study Group for their important help during the study.

The ProHOSP Study group included the following persons: U. Schild, K. Regez, R. Bossart, M. Wolbers, C. Blum, S. Neidert, H.C. Bucher, F. Mueller, J. Haeuptle, R. Zarbosky, R. Fiumefreddo, M. Wieland, C. Nusbaumer, A. Christ, R. Bingisser and K. Schneider (all University Hospital Basel, Basel, Switzerland) R. Thomann, R. Schoenenberger and R. Luginbuehl (all Buergerspital Solothurn, Solothurn, Switzerland); T. Fricker, C. Hoess, M. Krause, I. Lambinon and M. Zueger (all Kantonsspital Muensterlingen, Muensterlingen, Switzerland); C. Henzen and V. Briner (both Kantonsspital Luzern, Luzern, Switzerland); T. Bregenzer, D. Conen, A. Huber and Jody Staehelin (all Kantonsspital Aarau, Aarau, Switzerland); and C. Falconnier and C. Bruehlhardt (both Kantonsspital Liestal, Liestal, Switzerland).

\section{REFERENCES}

1 Niederman MS, Mandell LA, Anzueto A, et al. Guidelines for the management of adults with community-acquired pneumonia. Diagnosis, assessment of severity, antimicrobial therapy, and prevention. Am J Respir Crit Care Med 2001; 163: 1730-1754.

2 Woodhead M, Blasi F, Ewig S, et al. Guidelines for the management of adult lower respiratory tract infections. Eur Respir J 2005; 26: $1138-1180$.

3 Fine MJ, Auble TE, Yealy DM, et al. A prediction rule to identify low-risk patients with community-acquired pneumonia. New Engl J Med 1997; 336: 243-250.

4 Lim WS, van der Eerden MM, Laing R, et al. Defining community acquired pneumonia severity on presentation to hospital: an international derivation and validation study. Thorax 2003; 58: 377-382.

5 Schuetz P, Koller M, Christ-Crain M, et al. Predicting mortality with pneumonia severity scores: importance of model recalibration to local settings. Epidemiol Infect 2008; 136: 1628-1637.

6 Schuetz P, Christ-Crain M, Muller B. Procalcitonin and other biomarkers to improve assessment and antibiotic stewardship in infections - hope for hype? Swiss Med Wkly 2009; 139: 318-326.

7 Jensen JU, Heslet L, Jensen TH, et al. Procalcitonin increase in early identification of critically ill patients at high risk of mortality. Crit Care Med 2006; 34: 2596-2602.

8 Boussekey N, Leroy O, Alfandari S, et al. Procalcitonin kinetics in the prognosis of severe community-acquired pneumonia. Intensive Care Med 2006; 32: 469-472.

9 Haeuptle J, Zaborsky R, Fiumefreddo R, et al. Prognostic value of procalcitonin in Legionella pneumonia. Eur J Clin Microbiol Infect Dis 2009; 28: 55-60.

10 Muller B, Harbarth S, Stolz D, et al. Diagnostic and prognostic accuracy of clinical and laboratory parameters in communityacquired pneumonia. BMC Infect Dis 2007; 7: 10.

11 Kruger S, Ewig S, Marre R, et al. Procalcitonin predicts patients at low risk of death from community-acquired pneumonia across all CRB-65 classes. Eur Respir J 2008; 31: 349-355.

12 Huang DT, Weissfeld LA, Kellum JA, et al. Risk prediction with procalcitonin and clinical rules in community-acquired pneumonia. Ann Emerg Med 2008; 52: 48-58.

13 Schuetz P, Christ-Crain M, Wolbers M, et al. Procalcitonin guided antibiotic therapy and hospitalization in patients with lower respiratory tract infections: a prospective, multicenter, randomized controlled trial. BMC Health Serv Res 2007; 7: 102. 
14 Mandell LA, Wunderink RG, Anzueto A, et al. Infectious Diseases Society of America/American Thoracic Society consensus guidelines on the management of community-acquired pneumonia in adults. Clin Infect Dis 2007; 44: Suppl. 2, S27-S72.

15 Schuetz P, Albrich W, Christ-Crain M, et al. Procalcitonin for guidance of antibiotic therapy. Expert Rev Anti Infect Ther 2010; 5: 575-587.

16 Pencina MJ, D'Agostino RB Sr, D'Agostino RB Jr, et al. Evaluating the added predictive ability of a new marker: from area under the ROC curve to reclassification and beyond. Stat Med 2008; 27: 157-172.

17 Neill AM, Martin IR, Weir R, et al. Community acquired pneumonia: aetiology and usefulness of severity criteria on admission. Thorax 1996; 51: 1010-1016.

18 Bouadma L, Luyt CE, Tubach $\mathrm{F}$, et al. Use of procalcitonin to reduce patients' exposure to antibiotics in intensive care units (PRORATA trial): a multicentre randomised controlled trial. Lancet 2010; 375: 463-474.

19 Briel M, Schuetz P, Mueller B, et al. Procalcitonin-guided antibiotic use $v s$ a standard approach for acute respiratory tract infections in primary care. Arch Int Med 2008; 168: 2000-2007.

20 Schuetz P, Christ-Crain M, Wolbers M, et al. Effect of procalcitoninbased guidelines compared to standard guidelines on antibiotic use in lower respiratory tract infections: the randomized-controlled multicenter ProHOSP trial. JAMA 2009; 302: 1059-1066.

21 Christ-Crain M, Jaccard-Stolz D, Bingisser R, et al. Effect of procalcitonin-guided treatment on antibiotic use and outcome in lower respiratory tract infections: cluster-randomised, singleblinded intervention trial. Lancet 2004; 363: 600-607.

22 Christ-Crain M, Stolz D, Bingisser R, et al. Procalcitonin guidance of antibiotic therapy in community-acquired pneumonia: a randomized trial. Am J Respir Crit Care Med 2006; 174: 84-93.

23 Menendez R, Martinez R, Reyes S, et al. Biomarkers improve mortality prediction by prognostic scales in community-acquired pneumonia. Thorax 2009; 64: 587-591.

24 Menendez R, Martinez R, Reyes S, et al. Stability in communityacquired pneumonia: one step forward with markers? Thorax 2009; 64: 987-992.

25 Masia M, Gutierrez F, Shum C, et al. Usefulness of procalcitonin levels in community-acquired pneumonia according to the patients outcome research team pneumonia severity index. Chest 2005; 128: 2223-2229.

26 Muller F, Christ-Crain M, Bregenzer T, et al. Procalcitonin levels predict bacteremia in patients with community-acquired pneumonia: a prospective cohort trial. Chest 2010; 138: 121-129. 